\title{
VOLUME DEGENERACY OF THE TYPICAL CELL AND THE CHORD LENGTH DISTRIBUTION FOR POISSON-VORONOI TESSELLATIONS IN HIGH DIMENSIONS
}

\author{
KASRA ALISHAHI *** AND \\ MOHSEN SHARIFITABAR, ${ }^{* * * *}$ Sharif University of Technology
}

\begin{abstract}
This paper is devoted to the study of some asymptotic behaviors of Poisson-Voronoi tessellation in the Euclidean space as the space dimension tends to $\infty$. We consider a family of homogeneous Poisson-Voronoi tessellations with constant intensity $\lambda$ in Euclidean spaces of dimensions $n=1,2,3, \ldots$ First we use the Blaschke-Petkantschin formula to prove that the variance of the volume of the typical cell tends to 0 exponentially in dimension. It is also shown that the volume of intersection of the typical cell with the co-centered ball of volume $u$ converges in distribution to the constant $\lambda^{-1}\left(1-\mathrm{e}^{-\lambda u}\right)$. Next we consider the linear contact distribution function of the Poisson-Voronoi tessellation and compute the limit when the space dimension goes to $\infty$. As a by-product, the chord length distribution and the geometric covariogram of the typical cell are obtained in the limit.

Keywords: Poisson-Voronoi tessellation; typical cell; Blaschke-Petkantschin formula; high dimension; chord length distribution; linear contact distribution; geometric covariogram
\end{abstract}

2000 Mathematics Subject Classification: Primary 60D05

Secondary 60F25; 60F05

\section{Introduction}

Random tessellations are objects of interest in stochastic geometry both for theoretical reasons and for applications as models in material sciences, biology, geology, communication theory, and data analysis. One of the most important types of random tessellations, PoissonVoronoi tessellations, arises as a natural combination of Voronoi tessellations and Poisson point processes. Poisson-Voronoi tessellations appear to be good models in many applications, and although simpler than other models, the computation of many of its relevant statistical characteristics seems to be very difficult. There are many references on the subject of PoissonVoronoi tessellations; see, for example, [3], [6, pp. 455-459], [7], [8], [9], [10], [11], [12], [13], [15, pp. 291-410], and [17, pp. 307-338].

Using different approaches, Miles [8] and Møller [13] computed the mean value of a class of important geometric characteristics, known as intrinsic volumes, in arbitrary dimensions. Moreover, Møller introduced some stereological investigations based on simulation results. Okabe [15] provided computer simulation results for, in particular, two and three dimensions.

Received 7 February 2008; revision received 6 October 2008.

* Postal address: Department of Mathematics, Sharif University of Technology, Tehran, Iran.

** Email address: kasraalishahi@math.sharif.edu

*** Email address: sharifitabar@math.sharif.edu 
Muche and Stoyan [14], based on the results of Gilbert [2], derived integral formulae for the linear and spherical contact distribution functions in the case of the random set given by the union of all cell faces $(n=3)$ or edges $(n=2)$. The formulae are numerically tractable and lead to formulae for the chord length distribution function.

Although Poisson-Voronoi tessellations have received considerable attention in stochastic geometry, the field of tessellations in high dimensions is rather new and unexplored.

In this paper we take the first step towards investigating Poisson-Voronoi tessellations in high dimensions. We are especially interested in the limiting behavior of the volume and shape of a typical cell as the space dimension tends to $\infty$.

In Section 2 we discuss some preliminaries about Poisson-Voronoi tessellation, mean values, and the typical cell. In Section 3 we use the Blaschke-Petkantschin formula to prove that the variance of the $n$-volume of a typical cell of a Poisson-Voronoi tessellation with constant intensity $\lambda$ in $\mathbb{R}^{n}$ decreases to 0 as $n$ tends to $\infty$. We also prove that the convergence occurs exponentially, and we compute the exponent of convergence. In Section 4 we extend the result in a more geometrical form, and we show that the volume of the intersection of a typical cell with a co-centered ball of volume $u$ tends to the constant $\lambda^{-1}\left(1-\mathrm{e}^{-\lambda u}\right)$ as the dimension increases. This might be a significant result towards understanding the limiting behavior of the shapes of cells and might be interpreted as evidence for the fact that cells in highdimensional Poisson-Voronoi tessellations are somehow 'stretched'; but, owing to the measure concentration phenomena in high dimensions, we should be careful when giving geometric interpretations. In Section 5 we adopt a different mathematical quantification of the limiting shape behavior of cells by considering the linear contact distribution function. We will compute this function and, equivalently, the geometric covariogram associated to the typical cell in the limiting case of the space dimension going to $\infty$. In Section 6, as a by-product, the chord length distribution function is also computed in the limit. An efficient algorithm is also implemented to simulate the intersection of a line and a Poisson-Voronoi tessellation. The simulation results are compared with the analytic formula and some observations are discussed.

\section{Preliminaries}

A 'tessellation' (or a mosaic) in the Euclidean space $\mathbb{R}^{n}$ is a locally finite aggregate of space filling and nonoverlapping $n$-dimensional particles called cells, where the cells are convex compact sets with disjoint interiors. A 'random tessellation' $X$ of $\mathbb{R}^{n}$ is a random process with tessellations of $\mathbb{R}^{n}$ as realizations. A random tessellation $X$ is said to be 'stationary' if all translations of $X$ have the same distribution and is said to be 'isotropic' if all rotations of $X$ about the origin have the same distribution. A random tessellation which is both stationary and isotropic is called 'motion invariant'.

For a stationary random tessellation $X$ in $\mathbb{R}^{n}$, the mean $n$-volume of cells, the mean $(n-1)$-volume of their boundaries, and the mean values of other geometric characteristics are of interest. To define the mean value of an arbitrary characteristic on cells of $X$, we can average the values of the characteristic on all cells in a bounded domain of space and then enlarge this domain to the whole space. Although the above procedure of averaging does not refer to any particular cell of $X$, it is nonetheless possible to construct a (virtual) random cell called a 'typical cell' of $X$ which is denoted by $C_{t}$ such that the mean values computed as above are equal to the expectation of the characteristic on $C_{t}$.

A 'Voronoi tessellation' corresponding to a discrete set of points $\Gamma \subseteq \mathbb{R}^{n}$ is defined as $\left\{C_{x}(\Gamma): x \in \Gamma\right\}$, where $C_{x}$, the Voronoi cell of $\Gamma$ centered at $x$, is defined by $C_{x}=C_{x}(\Gamma)=$ $\left\{z \in \mathbb{R}^{n}:|z-x|=d(z, \Gamma)\right\}$. 
Voronoi tessellations corresponding to point processes are important examples of random tessellations. When the underlying point process $\tilde{X}$ is a homogeneous Poisson point process of intensity $\lambda$, the resulting random tessellation is named a 'Poisson-Voronoi tessellation' with intensity $\lambda$. Poisson-Voronoi tessellations are motion invariant, since Poisson point processes are. Miles and Maillardet [10] observed that, for the Poisson-Voronoi tessellation, a typical cell can be explicitly described by $C_{t}:=C_{0}(\tilde{X} \cup\{0\})$, that is, the Voronoi cell centered at the origin after adding the origin to the Poisson point process.

Sometimes it is useful to focus on $\hat{X}$, the union of all cell boundaries of a random tessellation, as a random set. Doing so, we can speak of the contact distribution function $H_{B}$ associated to convex compact sets $B$, defined by

$$
H_{B}(r)=\mathrm{P}(\hat{X} \cap r B \neq \varnothing) .
$$

The case where $B$ is a unit segment is of special interest. The contact distribution function in this case is called the 'linear contact distribution function' and is denoted by $H_{1}$. Obviously, when the random set is motion invariant, $H_{1}$ does not depend on the direction of the segment. It is well known that the linear contact distribution function is strongly connected to another important characteristic, namely, the 'chord length distribution function'. Consider the intersection between an arbitrary line and the random set $\hat{X}$, obtained, as above, from the cell faces of a motion invariant random tessellation. The chord length distribution function $L(r)$ is defined as the distribution function of the length of an interval which is (loosely speaking) uniformly chosen among these intervals, and the mean chord length $\bar{l}$ is the expected length of such a 'typical interval'. It can be proved [17] that

$$
H_{1}(r)=\left(\frac{1}{\bar{l}}\right) \int_{0}^{r}(1-L(t)) \mathrm{d} t .
$$

\section{Volume of the typical cell in high dimensions}

In this section we want to find the distribution of the volume of a typical cell of a PoissonVoronoi tessellation in high dimensions. Denoting by $V_{n}$ the $n$-volume of $C_{t}$ and representing $V_{n}$ as the integral of a characteristic function of the typical cell, we easily obtain the following formula for the moments of $V_{n}$ :

$$
\mathrm{E}\left(V_{n}^{k}\right)=\int_{\left(\mathbb{R}^{n}\right)^{k}} \exp \left[-\lambda \mu_{n}\left(\bigcup_{i=1}^{k} B\left(x_{i}\right)\right)\right] \mathrm{d} x_{1} \cdots \mathrm{d} x_{k},
$$

where $B\left(x_{i}\right)$ is the ball centered at $x_{i}$ with radius $\left|x_{i}\right|$ and $\mu_{n}$ refers to the Lebesgue measure in $\mathbb{R}^{n}$. The idea is to use this equality to find the moments as the dimension increases. It is a simple matter to prove some general estimates on the moments.

Lemma 3.1. For all $n$ and $k$,

$$
\frac{1}{\lambda^{k}} \leq \mathrm{E}\left(V_{n}^{k}\right) \leq \frac{k !}{\lambda^{k}}
$$

In particular,

$$
\mathrm{E}\left(V_{n}\right)=\frac{1}{\lambda}, \quad \frac{1}{\lambda^{2}} \leq \mathrm{E}\left(V_{n}^{2}\right) \leq \frac{2}{\lambda^{2}} .
$$


Proof. Note that, for any $x_{1}, \ldots, x_{k} \in \mathbb{R}^{n}$,

$$
\sum_{i=1}^{k} \mu_{n}\left(B\left(x_{i}\right)\right) \geq \mu_{n}\left(\bigcup_{i=1}^{k} B\left(x_{i}\right)\right) \geq \max _{1 \leq i \leq k} \mu_{n}\left(B\left(x_{i}\right)\right) .
$$

Therefore, using (3.1), we obtain

$$
\begin{aligned}
\int_{\left(\mathbb{R}^{n}\right)^{k}} \exp \left[-\lambda \sum_{i=1}^{k} \mu_{n}\left(B\left(x_{i}\right)\right)\right] \mathrm{d} x_{1} \cdots \mathrm{d} x_{k} & \leq \mathrm{E}\left(V_{n}^{k}\right) \\
& \leq \int_{\left(\mathbb{R}^{n}\right)^{k}} \exp \left[-\lambda \max _{1 \leq i \leq k} \mu_{n}\left(B\left(x_{i}\right)\right)\right] \mathrm{d} x_{1} \cdots \mathrm{d} x_{k} .
\end{aligned}
$$

Writing down the above integrals in polar coordinates,

$$
x_{i}=r_{i} \theta_{i}, \quad r_{i} \in[0, \infty), \quad \theta_{i} \in S^{n-1},
$$

and noting that $\mathrm{d} x_{i}=r_{i}^{n-1} \mathrm{~d} r_{i} \mathrm{~d} \theta_{i}$, the first integral becomes

$$
\begin{aligned}
\int_{\left(\mathbb{R}^{n}\right)^{k}} \exp \left[-\lambda \sum_{i=1}^{k} \mu_{n}\left(B\left(x_{i}\right)\right)\right] \mathrm{d} x_{1} \cdots \mathrm{d} x_{k} & =\left(\int_{\mathbb{R}^{n}} \exp \left[-\lambda \mu_{n}(B(x))\right] \mathrm{d} x\right)^{k} \\
& =\left(\int_{0}^{\infty} \int_{S^{n-1}} \exp \left[-\lambda \kappa_{n} r^{n}\right] r^{n-1} \mathrm{~d} \theta \mathrm{d} r\right)^{k} \\
& =\left(\int_{0}^{\infty} \exp \left[-\lambda \kappa_{n} r^{n}\right] r^{n-1}\left(n \kappa_{n}\right) \mathrm{d} r\right)^{k} \\
& =\left(\frac{1}{\lambda}\right)^{k},
\end{aligned}
$$

where $\kappa_{n}$ denotes the volume of the unit ball in $\mathbb{R}^{n}$ and, hence, $n \kappa_{n}$ is the $(n-1)$-area of $S^{n-1}$. The right-hand side of the inequality becomes

$$
\begin{aligned}
\int_{\left(\mathbb{R}^{n}\right)^{k}} & \exp \left[-\lambda \max _{1 \leq i \leq k} \mu_{n}\left(B\left(x_{i}\right)\right)\right] \mathrm{d} x_{1} \cdots \mathrm{d} x_{k} \\
= & \int_{[0, \infty)^{k}} \int_{\left(S^{n-1}\right)^{k}} \exp \left[-\lambda \kappa_{n} \max _{1 \leq i \leq k} r_{i}^{n}\right]\left(r_{1} \cdots r_{k}\right)^{n-1} \mathrm{~d} \theta_{1} \cdots \mathrm{d} \theta_{k} \mathrm{~d} r_{1} \cdots \mathrm{d} r_{k} \\
= & k \int_{r_{k}=\max _{1 \leq i \leq k}\left\{r_{i}\right\}} \int_{\left(S^{n-1}\right)^{k}} \exp \left[-\lambda \kappa_{n} r_{k}^{n}\right]\left(r_{1} \cdots r_{k}\right)^{n-1} \mathrm{~d} \theta_{1} \cdots \mathrm{d} \theta_{k} \mathrm{~d} r_{1} \cdots \mathrm{d} r_{k} \\
= & k \int_{0}^{\infty} \int_{\left[0, r_{k}\right]^{k-1}}\left(r_{1} \cdots r_{k-1}\right)^{n-1} \mathrm{~d} r_{1} \cdots \mathrm{d} r_{k-1}\left(n \kappa_{n}\right)^{k} \exp \left[-\lambda \kappa_{n} r_{k}^{n}\right] r_{k}^{n-1} \mathrm{~d} r_{k} \\
= & k \int_{0}^{\infty}\left(\int_{0}^{r_{k}} r^{n-1} \mathrm{~d} r\right)^{k-1}\left(n \kappa_{n}\right)^{k} \exp \left[-\lambda \kappa_{n} r_{k}^{n}\right] r_{k}^{n-1} \mathrm{~d} r_{k}
\end{aligned}
$$




$$
\begin{aligned}
& =k \int_{0}^{\infty}\left(\frac{r_{k}^{n}}{n}\right)^{k-1}\left(n \kappa_{n}\right)^{k} \exp \left[-\lambda \kappa_{n} r_{k}^{n}\right] r_{k}^{n-1} \mathrm{~d} r_{k} \\
& =k \lambda^{-k} \int_{0}^{\infty}\left(\lambda \kappa_{n} r_{k}^{n}\right)^{k-1} \exp \left[-\lambda \kappa_{n} r_{k}^{n}\right] n \lambda \kappa_{n} r_{k}^{n-1} \mathrm{~d} r_{k} \\
& =k \lambda^{-k} \int_{0}^{\infty} u^{k-1} \mathrm{e}^{-u} \mathrm{~d} u \\
& =k \lambda^{-k} \Gamma(k) \\
& =k ! \lambda^{-k} .
\end{aligned}
$$

This completes the proof.

In (3.1), both the integrand and the domain of integration vary with $n$, which makes it difficult to pass to the limit. Fortunately, the integrand depends only on the relative position of the $x_{i} \mathrm{~s}$. This strong symmetry helps to reduce the domain of integration by invoking the well-known formula of Blaschke [1] and Petkantschin [16] in stereology and integral geometry (see, e.g. [4, pp. 95-119], [8], [11], and [12]).

The Blaschke-Petkantschin formula is a geometric measure decomposition of the $q$-fold product of the Lebesgue measure in $\mathbb{R}^{n}$. Let $\mathcal{L}_{q}^{n}$ denote the space of all $q$-dimensional subspaces of $\mathbb{R}^{n}$, and let $\mathrm{d} L$ be the unique $O(n)$-invariant probability measure on $\mathcal{L}_{q}^{n}$. According to the Blaschke-Petkantschin formula for any integrable function $g:\left(\mathbb{R}^{n}\right)^{q} \rightarrow \mathbb{R}$,

$$
\begin{aligned}
\int_{\left(\mathbb{R}^{n}\right)^{q}} g\left(x_{1}, \ldots, x_{q}\right) \mathrm{d} x_{1}^{(n)} \cdots \mathrm{d} x_{q}^{(n)} \\
\quad=C_{n, q} \int_{\mathscr{L}_{q}^{n}} \int_{(L)^{q}} g\left(x_{1}, \ldots, x_{q}\right) \nabla_{q}\left(x_{1}, \ldots, x_{q}\right)^{n-q} \mathrm{~d} x_{1}^{(q)} \cdots \mathrm{d} x_{q}^{(q)} \mathrm{d} L,
\end{aligned}
$$

where $\nabla_{q}\left(x_{1}, \ldots, x_{q}\right)$ is $q$ ! times the $q$-dimensional measure of the $q$-simplex with vertices $0, x_{1}, \ldots, x_{q}$ and

$$
C_{n, q}=\frac{n !}{q !(n-q) !} \frac{\kappa_{n} \cdots \kappa_{n-q+1}}{\kappa_{q} \cdots \kappa_{1}} .
$$

The following theorem is our main result about the limiting behavior of Voronoi cells of a Poisson point process.

Theorem 3.1. There exists a constant $C$ independent of $n$ such that

$$
\operatorname{var}\left(V_{n}\right) \leq \frac{C}{\lambda^{2} \sqrt{n}}\left(\frac{4}{3 \sqrt{3}}\right)^{n}
$$

Proof. Applying the Blaschke-Petkantschin formula to (3.1) yields

$$
\begin{aligned}
\mathrm{E}\left(V_{n}^{2}\right) & =\int_{\left(\mathbb{R}^{n}\right)^{2}} \exp \left[-\lambda \mu_{n}(B(x) \cup B(y))\right] \mathrm{d} x \mathrm{~d} y \\
& =C_{n, 2} \int_{\left(\mathbb{R}^{2}\right)^{2}} \exp \left[-\lambda \mu_{n}(B(x) \cup B(y))\right] \nabla_{2}(x, y)^{n-2} \mathrm{~d} x \mathrm{~d} y .
\end{aligned}
$$

Using polar coordinates, $x=(r, \theta)$ and $y=(s, \psi)$, we obtain

$\mathrm{E}\left(V_{n}^{2}\right)=C_{n, 2} \int_{0}^{2 \pi} \int_{0}^{2 \pi} \int_{0}^{\infty} \int_{0}^{\infty} \exp \left[-\lambda \mu_{n}(B(x) \cup B(y))\right]|r s \sin (\theta-\psi)|^{n-2} r s \mathrm{~d} r \mathrm{~d} s \mathrm{~d} \theta \mathrm{d} \psi$. 


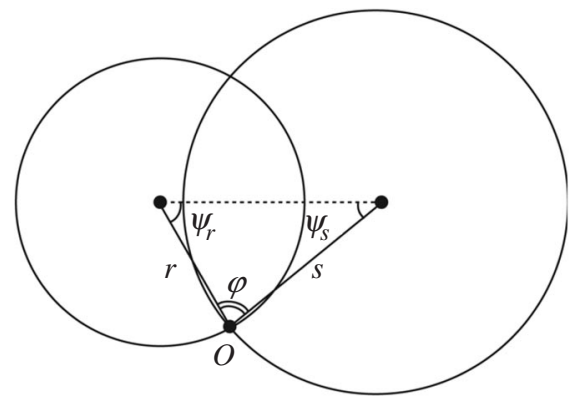

(a)

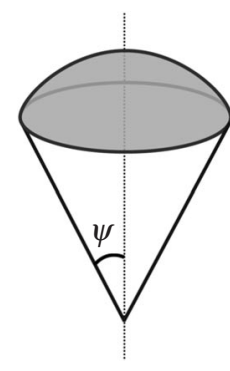

(b)

FIGURE 1: (a) Two intersected balls. (b) The cap of a ball.

But both $\mu_{n}(B(x) \cup B(y))$ and $\sin (\theta-\psi)$ depend only on $\varphi=\theta-\psi$ and, therefore,

$$
\mathrm{E}\left(V_{n}^{2}\right)=4 \pi C_{n, 2} \int_{0}^{\pi} \int_{0}^{\infty} \int_{0}^{\infty} \exp \left[-\lambda v_{n}(r, s, \varphi)\right](r s)^{n-1}(\sin \varphi)^{n-2} \mathrm{~d} r \mathrm{~d} s \mathrm{~d} \varphi,
$$

where $v_{n}(r, s, \varphi)$ stands for the $n$-volume of the union of two balls of radii $r$ and $s$ with angle $\varphi$ (see Figure 1(a)). The $n$-volume of a cap of a ball of radius $r$ with sweeping angle $\psi$ (see Figure 1(b)) is

$$
r^{n} \kappa_{n} \int_{0}^{\psi} \alpha_{n} \sin ^{n} \eta \mathrm{d} \eta
$$

where

$$
\alpha_{n}=\frac{\Gamma(n / 2+1)}{\Gamma((n+1) / 2) \Gamma(1 / 2)}
$$

normalizes $\sin ^{n} \varphi$ in $L^{1}(0, \pi)$ so that

$$
\int_{0}^{\pi} \alpha_{n} \sin ^{n} \varphi \mathrm{d} \varphi=1
$$

Hence,

$$
v_{n}(r, s, \varphi)=r^{n} \kappa_{n}+s^{n} \kappa_{n}-r^{n} \kappa_{n} \int_{0}^{\psi_{r}} \alpha_{n} \sin ^{n} \eta \mathrm{d} \eta-s^{n} \kappa_{n} \int_{0}^{\psi_{s}} \alpha_{n} \sin ^{n} \eta \mathrm{d} \eta,
$$

where $\psi_{r}$ and $\psi_{s}$ satisfy the following relations:

$$
\psi_{r}+\psi_{s}=\pi-\varphi, \quad r^{n} \sin ^{n} \psi_{r}=s^{n} \sin ^{n} \psi_{s} .
$$

Defining variables $R$ and $u$ by

$$
R=\left(r^{n}+s^{n}\right) \kappa_{n} \quad \text { and } \quad u=\frac{r^{n}}{r^{n}+s^{n}},
$$

we obtain $v_{n}(r, s, \varphi)=R F_{n}(u, \varphi)$, where

$$
F_{n}(u, \varphi):=1-u \int_{0}^{\psi_{r}} \alpha_{n} \sin ^{n} \eta \mathrm{d} \eta-(1-u) \int_{0}^{\psi_{s}} \alpha_{n} \sin ^{n} \eta \mathrm{d} \eta .
$$


Note that, since $\psi_{r}$ and $\psi_{s}$ are completely determined by only $u$ and $\varphi, F_{n}$ is actually a function of $u$ and $\varphi$. Since $r^{n} \kappa_{n}=R u$ and $s^{n} \kappa_{n}=R(1-u)$, the Jacobian of the transformation $(R, u) \rightarrow\left(r^{n} \kappa_{n}, s^{n} \kappa_{n}\right)$ is given by $\partial\left(r^{n} \kappa_{n}, s^{n} \kappa_{n}\right) / \partial(R, u)=R$. Therefore,

$$
\begin{aligned}
\mathrm{E}\left(V_{n}^{2}\right) & =4 \pi C_{n, 2} \int_{0}^{\pi} \int_{0}^{\infty} \int_{0}^{\infty} \exp \left[-\lambda v_{n}(r, s, \varphi)\right](r s)^{n-1}(\sin \varphi)^{n-2} \mathrm{~d} r \mathrm{~d} s \mathrm{~d} \varphi \\
& =\frac{4 \pi C_{n, 2}}{n^{2} \kappa_{n}^{2}} \int_{0}^{\pi} \int_{0}^{\infty} \int_{0}^{\infty} \exp \left[-\lambda v_{n}(r, s, \varphi)\right](\sin \varphi)^{n-2} \mathrm{~d}\left(r^{n} \kappa_{n}\right) \mathrm{d}\left(s^{n} \kappa_{n}\right) \mathrm{d} \varphi \\
& =\int_{0}^{\pi} \frac{4 \pi C_{n, 2}}{n^{2} \kappa_{n}^{2}} \sin ^{n-2} \varphi \int_{0}^{1} \int_{0}^{\infty} \exp \left[-\lambda R F_{n}(u, \varphi)\right] R \mathrm{~d} R \mathrm{~d} u \mathrm{~d} \varphi \\
& =\frac{1}{\lambda^{2}} \int_{0}^{\pi} \frac{4 \pi C_{n, 2}}{n^{2} \kappa_{n}^{2}} \sin ^{n-2} \varphi \int_{0}^{1} \frac{\mathrm{d} u}{F_{n}^{2}(u, \varphi)} \mathrm{d} \varphi .
\end{aligned}
$$

Substituting $\mu_{n}(B(x) \cup B(y))$ for $\mu_{n}(B(x))+\mu_{n}(B(y))$ in the right-hand side of (3.1) and proceeding as in Lemma 3.1, we obtain $1 / \lambda^{2}$. This is equivalent to replacing $F_{n}(u, \varphi)$ by 1 , which implies that $4 \pi C_{n, 2} / n^{2} \kappa_{n}^{2}=\alpha_{n-2}$. In fact, we can obtain this by straightforward calculations. Thus,

$$
\mathrm{E}\left(V_{n}^{2}\right)=\frac{1}{\lambda^{2}} \int_{0}^{\pi} \alpha_{n-2} \sin ^{n-2} \varphi \int_{0}^{1} \frac{\mathrm{d} u}{F_{n}^{2}(u, \varphi)} \mathrm{d} \varphi .
$$

Note that, since $F_{n}(u, \varphi)$ is the ratio of the volume of the union of two balls to the sum of their volumes, it is bounded between $\frac{1}{2}$ and 1 . The equations for $\psi_{r}$ and $\psi_{s}$ can be rewritten as

$$
\frac{\partial \psi_{r}}{\partial u}+\frac{\partial \psi_{s}}{\partial u}=0, \quad u \sin ^{n} \psi_{r}=(1-u) \sin ^{n} \psi_{s},
$$

which imply that

$$
\begin{aligned}
\frac{\partial F_{n}}{\partial u}= & -\int_{0}^{\psi_{r}} \alpha_{n} \sin ^{n} \eta \mathrm{d} \eta-u \frac{\partial \psi_{r}}{\partial u} \alpha_{n} \sin ^{n} \psi_{r} \\
& +\int_{0}^{\psi_{s}} \alpha_{n} \sin ^{n} \eta \mathrm{d} \eta-(1-u) \frac{\partial \psi_{s}}{\partial u} \alpha_{n} \sin ^{n} \psi_{s} \\
= & \int_{\psi_{r}}^{\psi_{s}} \alpha_{n} \sin ^{n} \eta \mathrm{d} \eta .
\end{aligned}
$$

This shows that, for each fixed $\varphi, F_{n}$ decreases on $\left(0, \frac{1}{2}\right)$ and increases on $\left(\frac{1}{2}, 1\right)$, and takes its minimum value at $u=\frac{1}{2}$. Therefore,

$$
\begin{aligned}
1-F_{n}(u, \varphi) & \leq 1-F_{n}\left(\frac{1}{2}, \varphi\right) \\
& =\int_{0}^{(\pi-\varphi) / 2} \alpha_{n} \sin ^{n} \eta \mathrm{d} \eta \\
& \leq \int_{0}^{(\pi-\varphi) / 2} \frac{\alpha_{n}}{\cos (\pi-\varphi) / 2} \cos \eta \sin ^{n} \eta \mathrm{d} \eta \\
& =\frac{\alpha_{n}}{(n+1) \sin \varphi / 2} \sin ^{n+1}\left(\frac{\pi-\varphi}{2}\right) \\
& =\frac{\alpha_{n} \cos ^{n+1}(\varphi / 2)}{(n+1) \sin \varphi / 2} .
\end{aligned}
$$


These facts imply that, for $n \geq 3$,

$$
\begin{aligned}
\lambda^{2} \mathrm{E}\left(V_{n}^{2}\right)-1 & =\int_{0}^{\pi} \alpha_{n-2} \sin ^{n-2} \varphi \int_{0}^{1}\left(\frac{1}{F_{n}^{2}(u, \varphi)}-1\right) \mathrm{d} u \mathrm{~d} \varphi \\
& \leq 8 \int_{0}^{\pi} \alpha_{n-2} \sin ^{n-2} \varphi \frac{\alpha_{n} \cos ^{n+1}(\varphi / 2)}{(n+1) \sin (\varphi / 2)} \mathrm{d} \varphi \\
& =\frac{2^{n+1} \alpha_{n-2} \alpha_{n}}{n+1} \int_{0}^{\pi} \sin ^{n-3}\left(\frac{\varphi}{2}\right) \cos ^{2 n-1}\left(\frac{\varphi}{2}\right) \mathrm{d} \varphi \\
& =\frac{2^{n+2} \alpha_{n-2} \alpha_{n}}{n+1} \int_{0}^{\pi / 2} \sin ^{n-3} \varphi \cos ^{2 n-1} \varphi \mathrm{d} \varphi \\
& =\frac{2^{n+1} \alpha_{n-2} \alpha_{n}}{n+1} \frac{\Gamma(n / 2-1) \Gamma(n)}{\Gamma(3 n / 2-1)} .
\end{aligned}
$$

Recalling that $\alpha_{n}=\Gamma(n / 2+1) / \Gamma((n+1) / 2) \Gamma(1 / 2)$ and using the Stirling inequality for the gamma function, the theorem follows after a straightforward calculation.

Corollary 3.1. The volume of the typical cell of the standard Poisson-Voronoi tessellation, $V_{n}$, converges in distribution to a constant value of $1 / \lambda$ as $n \rightarrow \infty$.

Remark 3.1. Using the Stirling inequality and numerical computations for small values of $n$, it is not difficult to show that we can choose $C=5$. Therefore, in the case of the standard Poisson-Voronoi tessellation (i.e. $\lambda=1$ ) we have $\operatorname{var}\left(V_{20}\right)<0.006$ and $\operatorname{var}\left(V_{100}\right)<3 \times 10^{-12}$, which is practically 0 .

Remark 3.2. The order of convergence of $V_{n}$ given in Theorem 3.1 is sharp in the sense that there exists a constant $C^{\prime}$ such that

$$
\operatorname{var}\left(V_{n}\right) \geq \frac{C^{\prime}}{\lambda^{2} \sqrt{n}}\left(\frac{4}{3 \sqrt{3}}\right)^{n}
$$

To show this, let $\varphi^{*}=\arcsin (1 / \sqrt{3})$ be the angle at which the function $\varphi \rightarrow \sin \varphi \cos (\varphi / 2)$ takes its maximum value $4 / 3 \sqrt{3}$, and let $\left(\varphi_{1}, \varphi_{2}\right)$ be a small interval containing $\varphi^{*}$ with the property that, for any $\varphi \in\left(\varphi_{1}, \varphi_{2}\right)$,

$$
\sin \varphi \cos \frac{\varphi}{2} \geq \frac{4}{3 \sqrt{3}}\left(1-v\left(\varphi-\varphi^{*}\right)^{2}\right),
$$

by a suitable choice of a positive constant $\nu$. As in previous calculations, for $\varphi \in\left(\varphi_{1}, \varphi_{2}\right)$ and $\frac{1}{4}<u<\frac{3}{4}$, from the inequality

$$
\int_{0}^{\psi} \alpha_{n} \sin ^{n} \eta \mathrm{d} \eta \geq \int_{0}^{\psi} \alpha_{n} \sin ^{n} \eta \cos \eta \mathrm{d} \eta=\frac{\alpha_{n} \sin ^{n+1} \psi}{n+1},
$$

we can write

$$
F_{n}(u, \varphi) \leq F_{n}\left(\frac{3}{4}, \varphi\right) \leq 1-\frac{3}{4} \frac{\alpha_{n} \sin ^{n+1} \psi_{r}}{n+1}-\frac{1}{4} \frac{\alpha_{n} \sin ^{n+1} \psi_{s}}{n+1} \leq 1-\frac{1}{4} \frac{\alpha_{n} \sin ^{n+1} \psi_{s}}{n+1},
$$

with $\psi_{r}+\psi_{s}=\pi-\varphi$ and $3 \sin ^{n} \psi_{r}=\sin ^{n} \psi_{s}$. This implies that $\sin \psi_{s} \geq \sin (\pi-\varphi) / 2=$ $\cos (\varphi / 2)$ and, hence,

$$
1-F_{n}(u, \varphi) \geq \frac{\alpha_{n}}{4(n+1)} \cos ^{n+1}\left(\frac{\varphi}{2}\right) \text {. }
$$


Now the previously mentioned bounds on $F$ and the Stirling inequality imply that

$$
\begin{aligned}
\lambda^{2} \operatorname{var}\left(V_{n}\right) & \geq \frac{3 \alpha_{n} \alpha_{n-2}}{16(n+1)} \int_{\varphi_{1}}^{\varphi_{2}} \sin ^{n-2} \varphi \cos ^{n+1}\left(\frac{\varphi}{2}\right) \mathrm{d} \varphi \\
& \geq C_{1} \int_{\varphi_{1}}^{\varphi_{2}} \sin ^{n} \varphi \cos ^{n}\left(\frac{\varphi}{2}\right) \mathrm{d} \varphi \\
& \geq C_{1}\left(\frac{4}{3 \sqrt{3}}\right)^{n} \int_{\varphi_{1}-\varphi^{*}}^{\varphi_{2}-\varphi^{*}}\left(1-v x^{2}\right)^{n} \mathrm{~d} x
\end{aligned}
$$

The change of variable $y=\sqrt{n} x$ shows that, for large $n$,

$$
\begin{aligned}
\lambda^{2} \operatorname{var}\left(V_{n}\right) & \geq \frac{C_{1}}{\sqrt{n}}\left(\frac{4}{3 \sqrt{3}}\right)^{n} \int_{\sqrt{n}\left(\varphi_{1}-\varphi^{*}\right)}^{\sqrt{n}\left(\varphi_{2}-\varphi^{*}\right)}\left(1-\frac{v y^{2}}{n}\right)^{n} \mathrm{~d} y \\
& \geq \frac{C_{2}}{\sqrt{n}}\left(\frac{4}{3 \sqrt{3}}\right)^{n} \int_{-1}^{1} \exp \left[-v y^{2}\right] \mathrm{d} y \\
& =\frac{C^{\prime}}{\sqrt{n}}\left(\frac{4}{3 \sqrt{3}}\right)^{n} .
\end{aligned}
$$

\section{How spherical is the typical cell in high dimensions?}

In the previous section we proved that, as the space dimension increases to $\infty$, the volumes of the cells become more and more similar. A natural question to ask in this context is whether the cells of a high-dimensional Poisson-Voronoi tessellation become similar in geometric shapes rather than just in volumes. If so, the first and most natural candidate for this limiting common shape is the ball. In this section we measure the distance between the typical cell and a ball co-centered with it. We have chosen the volume of the symmetric difference as the distance function. Let $B^{u}$ denote the ball centered at the origin with volume $u$, and let

$$
R_{n}(u)=\frac{\mu_{n-1}\left(C_{t} \cap \partial B^{u}\right)}{\mu_{n-1}\left(\partial B^{u}\right)} .
$$

Lemma 4.1. We have

$$
\mu_{n}\left(C_{t} \cap B^{u}\right)=\int_{0}^{u} R_{n}(v) \mathrm{d} v .
$$

Proof. Let $r_{u}$ be the radius of a ball with volume $u$, i.e. $\kappa_{n} r_{u}^{n}=u$. Then

$$
\begin{aligned}
\mu_{n}\left(C_{t} \cap B^{u}\right) & =\int_{B^{u}} \mathbf{1}_{C_{t}}(x) \mathrm{d} x \\
& =\int_{0}^{r_{u}} \int_{S^{n-1}} r^{n-1} \mathbf{1}_{C_{t}}(r y) \mathrm{d} y \mathrm{~d} r \\
& =\int_{0}^{u} \frac{1}{n \kappa_{n}} \int_{S^{n-1}} \mathbf{1}_{C_{t}}\left(r_{v} y\right) \mathrm{d} y \mathrm{~d} v \\
& =\int_{0}^{u} R_{n}(v) \mathrm{d} v .
\end{aligned}
$$

This completes the proof. 
Let $v$ be the probability measure defined by normalizing the $(n-1)$-dimensional Hausdorff measure on $\partial B^{u}$. We can represent the moments of $R_{n}(u)$ by integral expressions similar to those in (3.1) as follows.

Lemma 4.2. We have

$$
\mathrm{E}\left(R_{n}(u)^{k}\right)=\int_{\partial B^{u}} \cdots \int_{\partial B^{u}} \exp \left[-\lambda \mu_{n}\left(\bigcup_{i=1}^{k} B\left(x_{i}\right)\right)\right] v\left(\mathrm{~d} x_{1}\right) \cdots v\left(\mathrm{~d} x_{k}\right) .
$$

In particular,

$$
\mathrm{E}\left(R_{n}(u)\right)=\mathrm{e}^{-\lambda u}, \quad \mathrm{E}\left(R_{n}(u)^{2}\right)=\int_{\left(\partial B^{u}\right)^{2}} \exp \left[-\lambda \mu_{n}(B(x) \cup B(y))\right] v(\mathrm{~d} x) v(\mathrm{~d} y) .
$$

Proof. Clearly, $R_{n}(u)=\int_{\partial B^{u}} \mathbf{1}_{C_{t}}(x) v(\mathrm{~d} x)$ and, therefore,

$$
\begin{aligned}
\mathrm{E}\left(R_{n}(u)^{k}\right) & =\mathrm{E} \int_{\partial B^{u}} \cdots \int_{\partial B^{u}} \mathbf{1}_{C_{t}}\left(x_{1}\right) \cdots \mathbf{1}_{C_{t}}\left(x_{k}\right) v\left(\mathrm{~d} x_{1}\right) \cdots v\left(\mathrm{~d} x_{k}\right) \\
& =\int_{\partial B^{u}} \cdots \int_{\partial B^{u}} \mathrm{P}\left(x_{1}, \ldots, x_{k} \in C_{t}\right) v\left(\mathrm{~d} x_{1}\right) \cdots v\left(\mathrm{~d} x_{k}\right) \\
& =\int_{\partial B^{u}} \cdots \int_{\partial B^{u}} \exp \left[-\lambda \mu_{n}\left(\bigcup_{i=1}^{k} B\left(x_{i}\right)\right)\right] v\left(\mathrm{~d} x_{1}\right) \cdots v\left(\mathrm{~d} x_{k}\right) .
\end{aligned}
$$

Theorem 4.1. For any fixed $u, \mu_{n}\left(C_{t} \cap B^{u}\right)$ converges in $L^{2}$ and, consequently, in distribution to the constant $\lambda^{-1}\left(1-\mathrm{e}^{-\lambda u}\right)$ as $n$ tends to $\infty$.

\subsection{Proof of Theorem 4.1}

Note that, according to Lemmas 4.1 and 4.2,

$$
\begin{aligned}
\mathrm{E}\left(\mu_{n}\left(C_{t} \cap B^{u}\right)\right)=\mathrm{E}\left(\int_{0}^{u} R_{n}(v) \mathrm{d} v\right) & =\int_{0}^{u} \mathrm{E}\left(R_{n}(v)\right) \mathrm{d} v=\int_{0}^{u} \mathrm{e}^{-\lambda v} \mathrm{~d} v=\lambda^{-1}\left(1-\mathrm{e}^{-\lambda u}\right), \\
\operatorname{var}\left(\mu_{n}\left(C_{t} \cap B^{u}\right)\right) & =\operatorname{var}\left(\int_{0}^{u} R_{n}(v) \mathrm{d} v\right) \\
& =\mathrm{E}\left(\int_{0}^{u}\left(R_{n}(v)-\mathrm{e}^{-\lambda v}\right) \mathrm{d} v\right)^{2} \\
& \leq u \int_{0}^{u} \mathrm{E}\left(R_{n}(v)-\mathrm{e}^{-\lambda v}\right)^{2} \mathrm{~d} v \\
& =u \int_{0}^{u} \operatorname{var}\left(R_{n}(v)\right) \mathrm{d} v .
\end{aligned}
$$

Since the $R_{n}(v)$ s are uniformly bounded, by the Lebesgue dominated convergence theorem, it is sufficient to show the following lemma.

Lemma 4.3. $\lim _{n \rightarrow \infty} \operatorname{var}\left(R_{n}(v)\right)=0$.

Proof. By Lemma 4.2,

$$
\mathrm{E}\left(R_{n}(v)^{2}\right)=\int_{\left(\partial B^{v}\right)^{2}} \exp \left[-\lambda \mu_{n}(B(x) \cup B(y))\right] v(\mathrm{~d} x) v(\mathrm{~d} y) .
$$


Note that we can fix $x$ in the above integral because of the symmetry. The integrand depends only on $\varphi$, the angle between $x$ and $y$. More precisely, it is equal to $\exp \left[-2 \lambda v F_{n}\left(\frac{1}{2}, \varphi\right)\right]$, where $F_{n}$ is defined as in the proof of Theorem 3.1. So, the above integral can be rewritten as an integral over $\varphi$ only. Since the $(n-1)$-volume of the strip on $\partial B^{v}$ between $\varphi$ and $\varphi+d \varphi$ is proportional to $\sin ^{n-2} \varphi$, we conclude that

$$
\mathrm{E}\left(R_{n}(v)^{2}\right)=\int_{0}^{\pi} c_{n} \sin ^{n-2} \varphi \exp \left[-2 \lambda v F_{n}\left(\frac{1}{2}, \varphi\right)\right] \mathrm{d} \varphi .
$$

Since $v$ is a probability measure, so is $c_{n} \sin ^{n-2} \varphi \mathrm{d} \varphi$ (in fact, $c_{n}=\alpha_{n-2}$ by our notation used in proof of Theorem 3.1). Since $\frac{1}{2} \leq F_{n} \leq 1$ and $\mathrm{E}\left(R_{n}(v)\right)=\mathrm{e}^{-\lambda v}$ by Lemma 4.2, using the mean value theorem, we have

$$
\begin{aligned}
\operatorname{var}\left(R_{n}(v)\right) & =\int_{0}^{\pi} \alpha_{n-2} \sin ^{n-2} \varphi\left(\exp \left[-2 \lambda v F_{n}\left(\frac{1}{2}, \varphi\right)\right]-\mathrm{e}^{-2 \lambda v}\right) \mathrm{d} \varphi \\
& \leq 2 \lambda v \mathrm{e}^{-\lambda v} \int_{0}^{\pi} \alpha_{n-2} \sin ^{n-2} \varphi\left(1-F_{n}\left(\frac{1}{2}, \varphi\right)\right) \mathrm{d} \varphi \\
& =2 \lambda v \mathrm{e}^{-\lambda v} \int_{0}^{\pi} \alpha_{n-2} \sin ^{n-2} \varphi \int_{0}^{(\pi-\varphi) / 2} \alpha_{n} \sin ^{n} \eta \mathrm{d} \eta \mathrm{d} \varphi \\
& \leq C^{\prime \prime} \lambda v \mathrm{e}^{-\lambda v} \frac{1}{\sqrt{n}}\left(\frac{4}{3 \sqrt{3}}\right)^{n} .
\end{aligned}
$$

The last step is exactly as in the proof of Theorem 3.1.

\section{The linear contact distribution function}

In this section we focus on the linear contact distribution function as another tool that helps us to extract useful information from a random tessellation. As mentioned in the preliminaries, the linear contact distribution function for a motion invariant random tessellation (and especially for a Poisson-Voronoi tessellation) measures the probability that a given segment hits the boundary of some cells. Gilbert [2] proved the following double integral representation formula for the linear contact distribution function in the case of a Poisson-Voronoi tessellation with intensity $\lambda$ in $\mathbb{R}^{n}$ :

$$
H_{1}(r)=1-(n-1) \kappa_{n-1} \lambda \int_{0}^{\infty} \int_{0}^{\pi} \rho^{n-1} \sin ^{n-2} \varphi \exp \left[-\lambda \mu_{n}\left(K_{\rho, \varphi, r}\right)\right] \mathrm{d} \varphi \mathrm{d} \rho,
$$

where $K_{\rho, \varphi, r}$ is the union of two balls with radii $\rho$ and $\sqrt{\rho^{2}-2 \rho r \cos \varphi+r^{2}}$, and distance $r$ between their centers. Muche and Stoyan [14] used this formula to carry out some computations for dimensions $n=2$ and $n=3$. In this section we are going to pass to the limiting case, $n \rightarrow \infty$. First, we rewrite (5.1) in terms of new, appropriately defined variables

$$
t=\cot \varphi \quad \text { and } \quad y=\rho \sin \varphi,
$$

and define $G(y, t, r):=K_{\rho, \varphi, r}$. Doing so, we obtain

$$
\begin{aligned}
H_{1}(r) & =1-(n-1) \kappa_{n-1} \lambda \int_{-\infty}^{\infty} \int_{0}^{\infty} y^{n-1} \exp \left[-\lambda \mu_{n}\left(K_{\rho, \varphi, r}\right)\right] \mathrm{d} y \mathrm{~d} t \\
& =1-(n-1) \kappa_{n-1} \lambda \int_{-\infty}^{\infty} \int_{0}^{\infty} y^{n-1} \exp \left[-\lambda \mu_{n}(G(y, t, r))\right] \mathrm{d} y \mathrm{~d} t .
\end{aligned}
$$


Note that $H_{1}$ can be interpreted as a distribution function, and so we can consider its $j$ th moment,

$$
\begin{aligned}
M_{j}^{(n)} & =\int_{0}^{\infty} j r^{j-1}\left(1-H_{1}(r)\right) \mathrm{d} r \\
& =(n-1) \kappa_{n-1} \lambda \int_{0}^{\infty} \int_{-\infty}^{\infty} \int_{0}^{\infty} j r^{j-1} y^{n-1} \exp \left[-\lambda \mu_{n}(G(y, t, r))\right] \mathrm{d} y \mathrm{~d} t \mathrm{~d} r \\
& =(n-1) \kappa_{n-1} \lambda \int_{0}^{\infty} \int_{-\infty}^{\infty} \int_{0}^{\infty} j s^{j-1} y^{j+n-1} \exp \left[-\lambda \mu_{n}(G(y, t, s y))\right] \mathrm{d} y \mathrm{~d} t \mathrm{~d} s \\
& =(n-1) \kappa_{n-1} \lambda \int_{0}^{\infty} j s^{j-1} \int_{-\infty}^{\infty} \int_{0}^{\infty} y^{j+n-1} \exp \left[-\lambda y^{n} \mu_{n}(G(1, t, s))\right] \mathrm{d} y \mathrm{~d} t \mathrm{~d} s \\
& =\frac{(n-1) \kappa_{n-1}}{n \lambda^{j / n}} \int_{0}^{\infty} j s^{j-1} \int_{-\infty}^{\infty} \frac{\Gamma(1+j / n)}{\mu_{n}(G(1, t, s))^{1+j / n}} \mathrm{~d} t \mathrm{~d} s \\
& =\frac{(n-1) \kappa_{n-1} \Gamma(1+j / n)}{n^{(j+3) / 2} \kappa_{n}^{1+j / n} \lambda^{j / n}} \int_{0}^{\infty} j s^{j-1} \int_{-\infty}^{\infty} \frac{\mathrm{d} t \mathrm{~d} s}{\mu_{n}^{\prime}(G(1, t / \sqrt{n}, s / \sqrt{n}))^{1+j / n}},
\end{aligned}
$$

where $\mu_{n}^{\prime}=\left(1 / \kappa_{n}\right) \mu_{n}$, the Lebesgue measure normalized so that the measure of the unit ball is equal to 1 . Since $\kappa_{n}=\pi^{n / 2} / \Gamma(n / 2+1)$, according to Stirling's formula, the coefficient tends to $1 / \sqrt{2 \pi}(\sqrt{2 \pi \mathrm{e}})^{j}$ as $n$ tends to $\infty$. The union of the balls in the denominator can be expressed as the disjoint union of two caps (similar to Figure 1), and it is easy to see that the hyperplane which separates the two caps is perpendicular to the line joining the centers of the balls at the coordinate $t / \sqrt{n}$; hence, the denominator can be computed as the following integral expressions:

$$
\begin{aligned}
\mu_{n}^{\prime}\left(G\left(1, \frac{t}{\sqrt{n}}, \frac{s}{\sqrt{n}}\right)\right)= & \frac{\kappa_{n-1}}{\kappa_{n}} \int_{-\sqrt{1+t^{2} / n}}^{t / \sqrt{n}}\left(1+\frac{t^{2}}{n}-x^{2}\right)^{(n-1) / 2} \mathrm{~d} x \\
& +\frac{\kappa_{n-1}}{\kappa_{n}} \int_{t / \sqrt{n}}^{\sqrt{1+t^{2} / n}}\left(1+\frac{(t-s)^{2}}{n}-(x-s)^{2}\right)^{(n-1) / 2} \mathrm{~d} x \\
= & \frac{\kappa_{n-1}}{\kappa_{n}} \int_{-}^{t / \sqrt{n}} \sqrt{1+t^{2} / n}\left(1+\frac{t^{2}}{n}-x^{2}\right)^{(n-1) / 2} \mathrm{~d} x \\
& +\frac{\kappa_{n-1}}{\kappa_{n}} \int_{(t-s) / \sqrt{n}}^{\sqrt{1+(t-s)^{2} / n}}\left(1+\frac{(t-s)^{2}}{n}-x^{2}\right)^{(n-1) / 2} \mathrm{~d} x \\
= & \frac{\kappa_{n-1}}{\kappa_{n}}\left(1+\frac{t^{2}}{n}\right)^{n / 2} \int_{-1}^{t / \sqrt{n+t^{2}}}\left(1-x^{2}\right)^{(n-1) / 2} \mathrm{~d} x \\
& +\frac{\kappa_{n-1}}{\kappa_{n}}\left(1+\frac{(t-s)^{2}}{n}\right)^{n / 2} \int_{(t-s) / \sqrt{n+(t-s)^{2}}}^{1}\left(1-x^{2}\right)^{(n-1) / 2} \mathrm{~d} x \\
= & \frac{\kappa_{n-1}}{\kappa_{n} \sqrt{n}}\left(1+\frac{t^{2}}{n}\right)^{n / 2} \int_{-\sqrt{n}}^{t \sqrt{n} / \sqrt{n+t^{2}}}\left(1-\frac{x^{2}}{n}\right)^{(n-1) / 2} \mathrm{~d} x \\
& +\frac{\kappa_{n-1}}{\kappa_{n} \sqrt{n}\left(1+\frac{(t-s)^{2}}{n}\right)^{n / 2}} \\
& \times \int_{(t-s) \sqrt{n} / \sqrt{n+(t-s)^{2}}}^{\sqrt{n}}\left(1-\frac{x^{2}}{n}\right)^{(n-1) / 2} \mathrm{~d} x .
\end{aligned}
$$


Write the first integral in the form of

$$
\int_{-\infty}^{\infty}\left(1-\frac{x^{2}}{n}\right)^{(n-1) / 2} \mathbf{1}_{\left[-\sqrt{n}, t \sqrt{n} / \sqrt{\left.n+t^{2}\right]}\right.}(x) \mathrm{d} x .
$$

The integrand converges pointwise to $\exp \left[-x^{2} / 2\right] \mathbf{1}_{(-\infty, t]}(x)$ and is dominated by

$$
\exp \left[\frac{-x^{2}}{n} \frac{n-1}{2}\right] \leq \exp \left[\frac{-x^{2}}{4}\right]
$$

which is integrable. A similar argument applies for the second integral. Since $\kappa_{n-1} / \kappa_{n} \sqrt{n}$ converges to $1 / \sqrt{2 \pi}$,

$$
\begin{aligned}
\mu_{n}^{\prime}\left(G\left(1, \frac{t}{\sqrt{n}}, \frac{s}{\sqrt{n}}\right)\right) \rightarrow & \exp \left[\frac{t^{2}}{2}\right] \int_{-\infty}^{t} \frac{1}{\sqrt{2 \pi}} \exp \left[\frac{-x^{2}}{2}\right] \mathrm{d} x \\
& +\exp \left[\frac{(t-s)^{2}}{2}\right] \int_{t-s}^{\infty} \frac{1}{\sqrt{2 \pi}} \exp \left[\frac{-x^{2}}{2}\right] \mathrm{d} x \\
= & \exp \left[\frac{t^{2}}{2}\right] \Phi(t)+\exp \left[\frac{(t-s)^{2}}{2}\right](1-\Phi(t-s)),
\end{aligned}
$$

where $\Phi$ is the 'standard normal cumulative distribution function'. All that remains is to show that the integrand is dominated by an integrable function in order to prove the convergence of the moments $M_{j}^{(n)}$. Since the union of the balls contains the one with the maximum radius, the denominator has the lower bound $\max \left\{1+t^{2} / n, 1+(t-s)^{2} / n\right\}^{(n+j) / 2}$, which is greater than $\max \left\{1+t^{2} / n, 1+(t-s)^{2} / n\right\}^{n / 2}$. But $(1+x / n)^{n}$ is increasing with respect to $n$ for any positive $x$ and, hence, for $n \geq j+2$, the integrand is dominated by

$$
\frac{j s^{j-1}}{\max \left\{1+t^{2} /(j+2), 1+(t-s)^{2} /(j+2)\right\}^{(j+2) / 2}},
$$

which is integrable since

$$
\begin{aligned}
\int_{0}^{\infty} & \int_{-\infty}^{\infty} \frac{s^{j-1} \mathrm{~d} t \mathrm{~d} s}{\max \left\{1+t^{2} /(j+2), 1+(t-s)^{2} /(j+2)\right\}^{(j+2) / 2}} \\
& =\int_{0}^{\infty} \int_{-\infty}^{s / 2} \frac{s^{j-1} \mathrm{~d} t \mathrm{~d} s}{\left(1+(t-s)^{2} /(j+2)\right)^{(j+2) / 2}}+\int_{0}^{\infty} \int_{s / 2}^{\infty} \frac{s^{j-1} \mathrm{~d} t \mathrm{~d} s}{\left(1+t^{2} /(j+2)\right)^{(j+2) / 2}} \\
& =2 \int_{0}^{\infty} \int_{s / 2}^{\infty} \frac{s^{j-1} \mathrm{~d} t \mathrm{~d} s}{\left(1+t^{2} /(j+2)\right)^{(j+2) / 2}} \\
& =2 \int_{0}^{1} \int_{s / 2}^{\infty} \frac{s^{j-1} \mathrm{~d} t \mathrm{~d} s}{\left(1+t^{2} /(j+2)\right)^{(j+2) / 2}}+2 \int_{1}^{\infty} \int_{s / 2}^{\infty} \frac{s^{j-1} \mathrm{~d} t \mathrm{~d} s}{\left(1+t^{2} /(j+2)\right)^{(j+2) / 2}} \\
& \leq 2 \int_{0}^{\infty} \frac{\mathrm{d} t}{\left(1+t^{2} /(j+2)\right)^{(j+2) / 2}+2(j+2)^{(j+2) / 2}} \int_{1}^{\infty} \int_{s / 2}^{\infty} \frac{s^{j-1} \mathrm{~d} t \mathrm{~d} s}{t^{j+2}} \\
& =2 \sqrt{j+2} \int_{0}^{\pi / 2} \int_{1}^{\infty} \frac{\cos ^{j} \eta \mathrm{d} \eta+\frac{2^{j+2}(j+2)^{(j+2) / 2}}{s^{2}}}{j+1} \\
& <+\infty .
\end{aligned}
$$


It is concluded that

$$
\begin{aligned}
\lim _{n \rightarrow \infty} M_{j}^{(n)}= & \frac{1}{(\sqrt{2 \pi \mathrm{e}})^{j}} \int_{0}^{\infty} j s^{j-1} \frac{1}{\sqrt{2 \pi}} \\
& \times \int_{-\infty}^{\infty} \frac{\mathrm{d} t \mathrm{~d} s}{\exp \left[t^{2} / 2\right] \Phi(t)+\exp \left[(t-s)^{2} / 2\right](1-\Phi(t-s))} \\
= & \int_{0}^{\infty} j r^{j-1} \frac{1}{\sqrt{2 \pi}} \\
& \quad \times \int_{-\infty}^{\infty} \frac{\mathrm{d} t}{\exp \left[t^{2} / 2\right] \Phi(t)+\exp \left[(t-r \sqrt{2 \pi \mathrm{e}})^{2} / 2\right](1-\Phi(t-r \sqrt{2 \pi \mathrm{e}}))} \mathrm{d} r .
\end{aligned}
$$

Now we are ready to prove the main result.

Theorem 5.1. We have

$$
\begin{aligned}
\lim _{n \rightarrow \infty} & H_{1}(r) \\
& =H_{1}^{\infty}(r) \\
& :=1-\frac{1}{\sqrt{2 \pi}} \int_{-\infty}^{\infty} \frac{\mathrm{d} t}{\exp \left[t^{2} / 2\right] \Phi(t)+\exp \left[(t-r \sqrt{2 \pi \mathrm{e}})^{2} / 2\right](1-\Phi(t-r \sqrt{2 \pi \mathrm{e}}))} .
\end{aligned}
$$

Proof. The convergence of the moments has been shown. Denoting the $j$ th moment of the distribution $H_{1}^{\infty}$ by $M_{j}$, according to the classical moment problem, it is enough to show that $\lim \sup \sqrt[j]{M_{j}} / j<+\infty$. We will prove that $\lim \sup \sqrt[j]{M_{j}} / j=0$.

The function $\exp \left[x^{2} / 2\right] \Phi(x)$ is increasing because

$$
\begin{aligned}
\sqrt{2 \pi} \exp \left[\frac{x^{2}}{2}\right] \Phi(x) & =\exp \left[\frac{x^{2}}{2}\right] \int_{-\infty}^{x} \exp \left[\frac{-u^{2}}{2}\right] \mathrm{d} u \\
& =\exp \left[\frac{x^{2}}{2}\right] \int_{-\infty}^{0} \exp \left[\frac{-(u+x)^{2}}{2}\right] \mathrm{d} u \\
& =\int_{-\infty}^{0} \exp \left[-u x-\frac{u^{2}}{2}\right] \mathrm{d} u
\end{aligned}
$$

and the integrand is obviously increasing with respect to $x$ for $u \leq 0$. Similarly, it can be seen that the function $\exp \left[x^{2} / 2\right](1-\Phi(x))$ is decreasing. As a consequence, we have the following inequality for $s \geq 0$ :

$$
\exp \left[\frac{t^{2}}{2}\right] \Phi(t)+\exp \left[\frac{(t-s)^{2}}{2}\right](1-\Phi(t-s)) \geq \max \left\{\exp \left[\frac{t^{2}}{2}\right], \exp \left[\frac{(t-s)^{2}}{2}\right]\right\}
$$

Hence,

$$
\begin{aligned}
\int_{-\infty}^{\infty} & \frac{\mathrm{d} t}{\exp \left[t^{2} / 2\right] \Phi(t)+\exp \left[(t-s)^{2} / 2\right] \Phi(t-s)} \\
& \leq \int_{-\infty}^{s / 2} \exp \left[-\frac{(t-s)^{2}}{2}\right] \mathrm{d} t+\int_{s / 2}^{\infty} \exp \left[-\frac{t^{2}}{2}\right] \mathrm{d} t \\
& =2 \sqrt{2 \pi}\left(1-\Phi\left(\frac{s}{2}\right)\right) .
\end{aligned}
$$




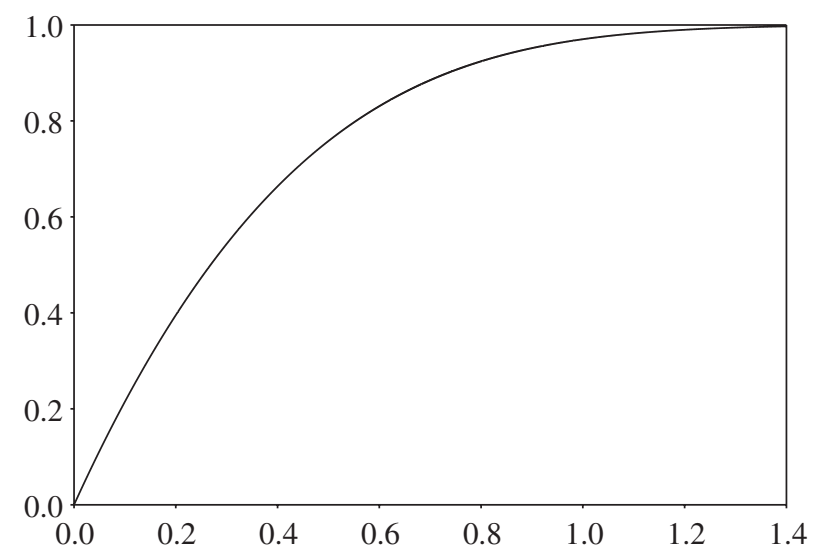

FIGURE 2: The limiting linear contact distribution function $H_{1}^{\infty}(r)$.

Therefore, using Stirling's inequality,

$$
\begin{aligned}
& M_{j} \leq \frac{2}{(\sqrt{2 \pi \mathrm{e}})^{j}} \int_{0}^{\infty} j s^{j-1}\left(1-\Phi\left(\frac{s}{2}\right)\right) \mathrm{d} s \\
& =\frac{2^{j+1}}{(\sqrt{2 \pi \mathrm{e}})^{j}} \int_{0}^{\infty} j s^{j-1}(1-\Phi(s)) \mathrm{d} s \\
& =\frac{2^{j+1}}{(\sqrt{2 \pi \mathrm{e}})^{j}} \int_{0}^{\infty} \frac{1}{\sqrt{2 \pi}} s^{j} \exp \left[-\frac{s^{2}}{2}\right] \mathrm{d} s \\
& =\frac{2^{j+1}}{(\sqrt{2 \pi \mathrm{e}})^{j} \sqrt{2 \pi}} \int_{0}^{\infty}(2 u)^{(j-1) / 2} \mathrm{e}^{-u} \mathrm{~d} u \\
& =\left(\frac{2}{\sqrt{\pi \mathrm{e}}}\right)^{j} \frac{\Gamma((j+1) / 2)}{\sqrt{\pi}} \\
& \leq C\left(\frac{2 j}{\pi \mathrm{e}^{2}}\right)^{j / 2},
\end{aligned}
$$

which completes the proof.

Figure 2 illustrates the limiting linear contact distribution function as computed in Theorem 5.1 .

Theorem 5.1 has a geometrical counterpart which is perhaps helpful in understanding the shapes of cells in a high-dimensional Poisson-Voronoi tessellation. For $K \subseteq \mathbb{R}^{n}$, the geometric covariogram of $K$ is the function $g_{K}: \mathbb{R}^{n} \rightarrow[0, \infty)$ defined as $g_{K}(h)=\mu_{n}(K \cap(K+h))$. The geometric covariogram can also be defined when $K$ is random by taking the expectation, i.e. $g_{K}(h)=\mathrm{E}\left(\mu_{n}(K \cap(K+h))\right)$. It is not difficult to prove that, for a stationary random tessellation, the linear contact distribution is related to the geometric covariogram of the typical cell $g_{C_{t}}$ by the following simple formula (see, e.g. [5, p. 136])

$$
1-H_{1}(r)=\frac{g_{C_{t}}(r)}{g_{C_{t}}(0)}
$$

In our case, $g_{C_{t}}(0)=\mathrm{E}\left(\mu_{n}\left(C_{t}\right)\right)=\lambda^{-1}$, and so we have the following result. 


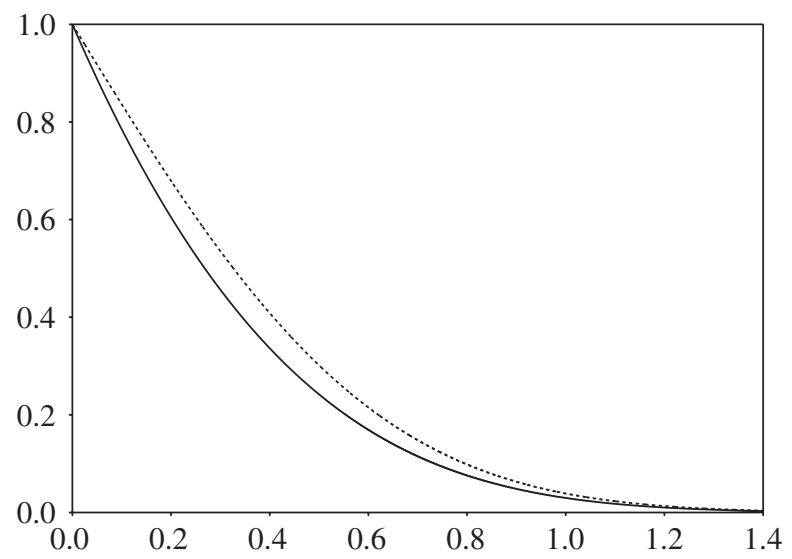

FIGURE 3: The solid and dotted lines correspond to the limiting geometric covariograms of $C_{t}$ and $B^{1 / \lambda}$, respectively. Here $\lambda=1$.

Corollary 5.1. If $g_{n}$ is the geometric covariogram of the typical cell of a homogeneous PoissonVoronoi tessellation in $\mathbb{R}^{n}$ with intensity $\lambda$,

$$
\lim _{n \rightarrow \infty} g_{n}(r)=\frac{1}{\lambda \sqrt{2 \pi}} \int_{-\infty}^{\infty} \frac{\mathrm{d} u}{\exp \left[t^{2} / 2\right] \Phi(t)+\exp \left[(t-r \sqrt{2 \pi \mathrm{e}})^{2} / 2\right](1-\Phi(t-r \sqrt{2 \pi \mathrm{e}}))} .
$$

It might be interesting at this point to compare the limiting geometric covariogram for the typical cell of a high-dimensional Poisson-Voronoi tessellation with the geometric covariogram of the high-dimensional ball of volume $\lambda^{-1}$. The latter can be computed in an almost straightforward way (write the intersection of the balls as a disjoint union of two caps and scale with $\sqrt{n})$ :

$$
\lim _{n \rightarrow \infty} g_{B^{1 / \lambda}}(r)=\frac{2}{\lambda} \Phi\left(-r \sqrt{\frac{\pi \mathrm{e}}{2}}\right) .
$$

Figure 3 illustrates the two numerically computed limiting geometric covariograms. It is remarkable that the two functions are close together.

In Section 4 we proved that if $B^{u}$ denotes the ball centered at the origin with volume $u$ then $\mu_{n}\left(C_{t}^{(n)} \cap B^{u}\right)$ converges in distribution to the constant $\lambda^{-1}\left(1-\mathrm{e}^{-\lambda u}\right)$ as $n$ tends to $\infty$. In other words, the volume of the intersection of the typical cell and the ball becomes deterministic in high dimensions. The geometric covariogram measures the expectation of the volume of the intersection of the typical cell with a translation of itself. Corollary 5.1 gives the value of this expectation in high dimensions. A natural question to ask is whether this volume becomes deterministic as in the case of the intersection with a ball of given volume when the dimension increases.

\section{The chord length distribution function}

Recall that the chord length distribution function for a random tessellation is the distribution of lengths of intervals formed by intersecting the tessellation with a fixed line. To use (2.1), which relates the linear contact distribution function and the chord length distribution function, 
it is necessary to know the value of $\bar{l}$, the mean chord length. Gilbert [2] provided the following formula:

$$
\bar{l}=\lambda^{-1 / n} \frac{n \Gamma((n+1) / 2)^{2} \Gamma(n-1 / 2)}{2 \Gamma(n) \Gamma(n / 2+1)^{2-1 / n} \Gamma(2-1 / n)} .
$$

Combining this formula with Theorem 5.1, which gives the limiting behavior of the linear contact distribution function, we can derive a similar asymptotic formula for the chord length distribution function as well.

Theorem 6.1. As $n$ increases to $\infty$, the chord length distribution functions converge to the following distribution function:

$$
\begin{aligned}
L^{\infty}(r):=1 & -\frac{1}{2 \sqrt{\pi \mathrm{e}}} \\
& \times \int_{-\infty}^{\infty} \frac{\partial}{\partial r}\left(\frac{1}{\exp \left[t^{2} / 2\right] \Phi(t)+\exp \left[(t-r \sqrt{2 \pi \mathrm{e}})^{2} / 2\right](1-\Phi(t-r \sqrt{2 \pi \mathrm{e}}))}\right) \mathrm{d} t .
\end{aligned}
$$

Proof. From (2.1),

$$
\int_{0}^{\infty} j r^{j-1}(1-L(r)) \mathrm{d} r=\int_{0}^{\infty} j r^{j-1} \bar{l} H_{l}^{\prime}(r) \mathrm{d} r=j \bar{l} M_{j-1}^{(n)} .
$$

Using (6.1) and Stirling's estimation of the gamma function, we conclude that

$$
\lim _{n \rightarrow \infty} \bar{l}=\frac{1}{\sqrt{2 \mathrm{e}}}
$$

Hence,

$$
\begin{aligned}
\lim _{n \rightarrow \infty} \int_{0}^{\infty} j r^{j-1}(1-L(r)) \mathrm{d} r & =\frac{j}{\sqrt{2 \mathrm{e}}} M_{j-1} \\
& =\frac{j}{\sqrt{2 \mathrm{e}}} \int_{0}^{\infty} r^{j-1} \frac{\mathrm{d} H_{1}^{\infty}}{\mathrm{d} r} \mathrm{~d} r \\
& =\int_{0}^{\infty} j r^{j-1}\left(1-L^{\infty}(r)\right) \mathrm{d} r
\end{aligned}
$$

So, again, the theorem is a consequence of the convergence of the moments and the estimation on the $M_{j} \mathrm{~s}$, which implies that

$$
\limsup \frac{\sqrt[j]{(} j / \sqrt{2 \mathrm{e}}) M_{j-1}}{j}=0<+\infty .
$$

Remark 6.1. Muche and Stoyan [14] numerically computed the densities of the chord length distribution functions $l_{n}(r)$ in $\mathbb{R}^{n}$ for $2 \leq n \leq 7$. They conjectured that 'the limit of $l_{n}(0)$ as $n \rightarrow \infty$ is very large'. Using Theorem 6.1, after some manipulations of the integrals containing the normal distribution function, we can compute the exact limiting value as $\lim _{n \rightarrow \infty} l_{n}(0)=\sqrt{2 \mathrm{e}}(\sqrt{3}-\pi / 3) \approx 1.5968$ (see Figure 4).

In the remaining part of this section we numerically calculate the chord length distribution function in arbitrary dimensions by computer simulation. The computation of the Voronoi tessellation for a given set of data points is a central and well-studied problem in computational 


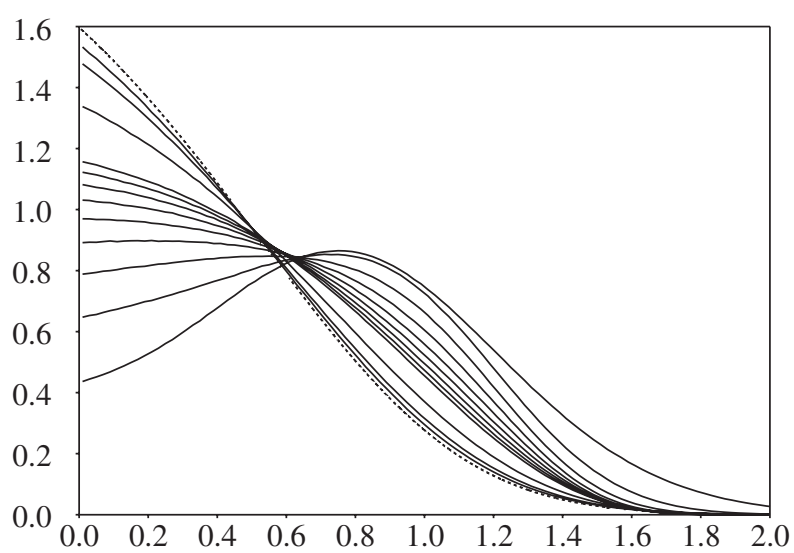

FIGURE 4: Chord length density from simulation results in dimensions 2, 3, 4, 5, 6, 7, 8, 9, 10, 20, 50, and 100. The dotted line is the analytic limiting chord length density. We ran the algorithm 100 times with $L=500000$ to obtain the histograms.

geometry. The complexity of this problem increases rapidly with dimension and, in more than two or three dimensions, the algorithms are not practically executable.

Fortunately, we are interested in the intersection of the Poisson-Voronoi tessellation with a line, and this intersection can be computed much more efficiently. We implement a tricky algorithm which helps perform simulations in very high dimensions.

Consider a (long) segment $\mathcal{L}$ with length $R+L+R$ and a cylinder with radius $R$ and axis $\mathcal{L}$. We use cylindrical coordinates to generate Poisson points inside the cylinder, i.e. let $N$ be a Poisson random variable with the mean value $(L+2 R) \kappa_{n-1} R^{n-1}$ and generate $N$ points with coordinates $(x, r)$ such that $x$ is uniformly distributed in $[-R, L+R], r$ is independent of $x$, and $r^{n-1}$ has uniform distribution on $\left[0, R^{n-1}\right]$. We want to specify the tessellation induced on $[0, L]$ for a realization of the Poisson point process. The radius $R$ should be large enough such that Poisson points outside the cylinder have no effect on the tessellation induced on $[0, L]$ (we will explain this more accurately later). Note that the cylindrical angle of points has no effect on the tessellation induced on $\mathcal{L}$. More precisely, $C_{z}$ is the intersection of half-spaces determined by mid-perpendicular hyperplanes between $z$ and all other Poisson points, each $(n-1)$-face of cells is the mid-perpendicular hyperplane of two Poisson points, and the intersection of $\mathcal{L}$ with the mid-perpendicular hyperplane does not depend on the cylindrical angle of the two points. In fact, if the coordinates of these two points are $\left(x_{1}, r_{1}\right)$ and $\left(x_{2}, r_{2}\right)$, the $x$-coordinate of the intersection is such that $\left(x-x_{1}\right)^{2}+r_{1}^{2}=\left(x-x_{2}\right)^{2}+r_{2}^{2}$, i.e. $x=\left(r_{2}^{2}+x_{2}^{2}-r_{1}^{2}-x_{1}^{2}\right) / 2\left(x_{2}-x_{1}\right)$. We may imagine that all things happen on the upper half-plane of $\mathbb{R}^{2}$, and we are interested in the tessellation induced on the boundary.

So first generate Poisson points and then sort these points according to their $x$-coordinates and call them $z_{1}, \ldots, z_{N}$. Let $x_{i+1 / 2}$ be the $x$-coordinate of the intersection of the mid-perpendicular hyperplane of $z_{i}$ and $z_{i+1}$ with the continuation of $\mathcal{L}$. The important fact is that a point with $x$-coordinate greater than $x_{i+1 / 2}$ on $\mathcal{L}$ is nearer to $z_{i+1}$ than $z_{i}$, and vice versa. Now the condition $x_{i+1 / 2}<x_{i-1 / 2}$ ensures that $C_{z_{i}}$ has no intersection with $\mathcal{L}$ (since, by the above fact, any point on $\mathcal{L}$ will be nearer to $z_{i-1}$ or $z_{i+1}$ than $z_{i}$ ) and, hence, we can omit such a point without any effect on the tessellation of $\mathscr{L}$. Omit all indices with this property, reindex 
the remaining points, and calculate the $x_{i+1 / 2} \mathrm{~s}$ again. Do this until no more points can be omitted. Again, the above fact implies that $C_{z_{i}} \cap \mathcal{L}=\left[x_{i-1 / 2}, x_{i+1 / 2}\right]$. It is simple to see that the maximum distance of any point on $\mathcal{L}$ from the set of $z_{i} \mathrm{~s}$ is $\max _{i}\left\{\sqrt{\left(x_{i}-x_{i+1 / 2}\right)^{2}+r_{i}^{2}}\right\}$, and if this is less than $R$, it means that farther points of the Poisson process (the Poisson points out of the cylinder) have no effect on $[0, L]$ and we are done. Otherwise, we should enlarge $R$ and generate more Poisson points. The pseudocode for this algorithm is as follows.

Algorithm 6.1. 1. Choose $L$ and $R$ large enough, and generate Poisson points on the cylinder with axis $R+L+R$ and radius $R$.

2. Sort the points with respect to their $x$-coordinate.

3. $x_{i+1 / 2}:=\left(r_{i+1}^{2}+x_{i+1}^{2}-r_{i}^{2}-x_{i}^{2}\right) / 2\left(x_{i+1}-x_{i}\right)$.

4. Omit the ith Poisson point if $x_{i+1 / 2}<x_{i-1 / 2}$. (Check this for all indices.)

5. Return to step 3 if any point is omitted.

6. If $\max \left\{\sqrt{\left(x_{i}-x_{i+1 / 2}\right)^{2}+r_{i}^{2}}\right\}>R$, enlarge $R$, generate farther Poisson points, and go back to step 2 .

7. The $x_{i+1 / 2}$ which are in $[0, L]$ specify the tessellation on this segment.

Figure 4 illustrates the distribution of chord lengths simulated by Algorithm 6.1.

Remark 6.2. Our experiments for dimensions $n \leq 10^{12}$ show that choosing $R$ such that $\kappa_{n-1} R^{n-1} \geq 32$ is practically enough. Also, the loop of omitting the points is repeated less than 11 times.

Remark 6.3. Simulation results in dimension $n=10^{12}$ indicate that the correlation between the lengths of consecutive intervals is negative, although small in absolute value. According to simulations, it seems that this correlation is negative in all dimensions and converges to approximately -0.118 as the space dimension goes to $\infty$.

\section{References}

[1] BlaschKe, W. (1935). Integralgeometrie 1. Ermittlung der Dichten für Linear Unterräume im $E_{n}$. Hermann, Paris.

[2] Gilbert, E. N. (1962). Random subdivisions of space into crystals. Ann. Math. Statist. 33, 958-972.

[3] Hug, D. (2007). Random mosaics. In Stochastic Geometry, ed. W. Weil, Springer, Berlin, pp. 247-266.

[4] Jensen, E. B. V. (1998). Local Stereology (Adv. Ser. Statist. Sci. Appl. Prob. 5). World Scientific, River Edge, NJ.

[5] Lantuejoul, C. (2002). Geostatistical Simulation: Models and Algorithms. Springer, Berlin.

[6] Mathai, A. M. (1999). An Introduction to Geometrical Probability. Gordon and Breach, Amsterdam.

[7] Meijering, J. L. (1953). Interface area, edge length, and number of vertices in crystal aggregates with random nucleation. Philips Res. Rep. 8, 270-290.

[8] Miles, R. E. (1974). A synopsis of 'Poisson flats in Euclidean spaces'. In Stochastic Geometry, eds E. F. Harding and D. G. Kendall. John Wiley, New York, pp. 202-227.

[9] Miles, R. E. (1984). Sectional Voronoi tessellations. Revista de la Unión Matemática Argentina 29, $310-327$.

[10] Miles, R. E. ANd Maillardet, R. J. (1982). The basic structures of Voronoi and generalized Voronoi polygons. In Essays in Statistical Science (J. Appl. Prob. Spec. Vol. 19A), eds J. Gani and E. J. Hannan, Applied Probability Trust, Sheffield, pp. 97-111.

[11] MøLler, J. (1994). Lectures on Random Voronoi Tessellations (Lecture Notes Statist. 87). Springer, New York.

[12] MøLler, J. (1987). A simple derivation of a formula of Blaschke and Petkantschin. Res. Rep. 138, Department of Theoretical Statistics, Institute of Mathematics, University of Aarhus.

[13] Møller, J. (1989). Random tessellations in $\mathbb{R}^{n}$. Adv. Appl. Prob. 21, 37-73. 
[14] Muche, L. and Stoyan, D. (1992). Contact and chord length distributions of the Poisson-Voronoi tessellation. J. Appl. Prob. 29, 467-471.

[15] OKabe, A., Boots, B., Sugihara, K. And Chiu, S. N. (2000). Spatial Tessellations: Concepts and Applications of Voronoi Diagrams, 2nd edn. John Wiley, Chichester.

[16] PetKantschin, B. (1936). Integralgeometrie 6. Zusammenhänge zwischen den Dichten der linearen Unterräume im n-dimensionalen Raum. Abh. Math. Seminar Hamburg 11, 249-310.

[17] Stoyan, D., Kendall, W. S. and Mecke, J. (1987). Stochastic Geometry and Its Applications. John Wiley, Chichester. 